\title{
Do infants represent the face in a viewpoint-invariant manner? Neural adaptation study as measured by near-infrared spectroscopy
}

\author{
Megumi Kobayashi ${ }^{1,2}{ }^{*}$, Yumiko Otsuka ${ }^{3,4}$, Emi Nakato ${ }^{5}$, So Kanazawa ${ }^{4}$, Masami K. Yamaguchi ${ }^{1}$ and \\ Ryusuke Kakigi ${ }^{5}$
}

${ }^{1}$ Department of Psychology, Chuo University, Hachioji, Tokyo, Japan

2 Japan Society for the Promotion of Sciences, Chiyoda Ward, Tokyo, Japan

${ }^{3}$ School of Psychology, The University of New South Wales, Sydney, NSW, Australia

${ }^{4}$ Department of Psychology, Japan Women's University, Kawasaki, Kanagawa, Japan

${ }^{5}$ Department of Integrative Physiology, National Institute for Physical Sciences, Okazaki, Aichi, Japan

\section{Edited by:}

Shuhei Yamaguchi, Shimane

University, Japan

Reviewed by:

Marlene Behrmann, Carnegie Mellon University, USA

Martin J. Herrmann, Universtity of

Würzburg, Germany

*Correspondence:

Megumi Kobayashi, Department of

Psychology, Chuo University, 742-1,

Higashinakano, Hachioji, Tokyo

192-0393, Japan.

e-mail:oc084001@grad.tamacc.

chuo-u.ac.jp
Recent adult functional magnetic resonance imaging (fMRI) studies reported that facesensitive cortical areas showed attenuated responses to the repeated presentation of an identical facial image compared to the presentation of different facial images (fMRIadaptation effects: e.g., Andrews and Ewbank, 2004). Building upon this finding, the current study, employing the adaptation paradigm, used near-infrared spectroscopy (NIRS) to explore the neural basis of face processing in infants. In Experiment 1, we compared hemodynamic responses in the bilateral temporal regions during the repeated presentation of the same face (the same-face condition) and the sequential presentation of different faces (the different-face condition). We found that (1) hemodynamic responses in the channels around the T5 and T6 regions increased during the presentation of different faces compared to those during the presentation of different objects; and that (2) these channels showed significantly lower response in the same-face condition than in the different-face condition, demonstrating the neural adaptation effect in 5- to 8-month-olds as measured by NIRS. In Experiment 2, when faces in both the same-face and different-face conditions were changed in viewpoint, lower hemodynamic responses in the same-face condition were found in 7- to 8-month-olds but not in 5- to 6-month-olds. Our results suggest that faces are represented in a viewpoint-invariant manner in 7- and 8-month-old infants.

Keywords: adaptation effect, face, infants, viewpoint-invariant, near-infrared spectroscopy, NIRS

\section{INTRODUCTION}

Near-infrared spectroscopy (NIRS) is a non-invasive neuroimaging method that measures changes in concentrations of oxyhemoglobin (oxy-Hb), deoxyhemoglobin (deoxy-Hb), and totalhemoglobin (total-Hb; Villringer and Chance, 1997; Hoshi et al., 2001). Previous studies conducting simultaneously measurement of NIRS and functional magnetic resonance imaging (fMRI) during the simple motor task revealed that there is a strong correlation between signals measured with NIRS and fMRI (Toronov et al., 2001, 2007; Strangman et al., 2002). In the recent study, Cui et al. (2011) also showed such correlation between NIRS and fMRI signals even for cognitive task. Therefore, NIRS is reliable method to measure the brain activation. Unlike fMRI and positron emission tomography (PET), NIRS can record even if the subjects' body and brain do not remain in fixed position. Hence, NIRS makes it possible to measure the brain activities of infants while they are awake.

Previous developmental studies of infants using NIRS have shown that NIRS can detect the brain activity induced by various visual patterns (Taga et al., 2003, 2004; Kusaka et al., 2004; Karen et al., 2008), odor stimulation (Bartocci et al., 2000), auditory stimulation (Taga and Asakawa, 2007), and speech patterns (Pena et al., 2003; Bortfeld et al., 2007). Furthermore, some studies have shown the brain activity that underlies infants' cognitive processing in tasks such as the perception of objects (Baird et al., 2002; Wilcox et al., 2005, 2008, 2009; Watanabe et al., 2010), and faces (Otsuka et al., 2007; Carlsson et al., 2008; Grossmann et al., 2008; Nakato et al., 2009, 2011a,b; Honda et al., 2010).

Otsuka et al. (2007) used NIRS to reveal an inter-hemispheric difference in infants' face processing between the presentation of upright and inverted faces. They revealed that the presentation of upright faces induces a greater concentration of oxy- $\mathrm{Hb}$ and total$\mathrm{Hb}$ in the right temporal area than the presentation of objects, suggesting that the right hemisphere is more important than the left for the processing of upright faces. Moreover, Nakato et al. (2009) revealed that developmental differences in infants' brain activity occur during the presentation of frontal and profile views, and suggest that the right temporal area is dominant for the profile as well as the frontal view. Consistent with Otsuka et al. (2007) and Nakato et al. (2009), more recent NIRS studies have reported that infants' temporal region is selectively activated by the presentation of faces using facial images of facial expressions (Nakato 
et al., 2011b), canonical vs. scrambled faces (Honda et al., 2010), dynamic point-lighted facial display (Ichikawa et al., 2010), mutual gaze vs. averted gaze (Grossmann et al., 2008), and mother's face vs. strangers' faces (Carlsson et al., 2008; Nakato et al., 2011a). These previous NIRS studies of infants showed that the temporal area was involved in the face recognition during infancy (Otsuka et al., 2007; Carlsson et al., 2008; Grossmann et al., 2008; Nakato et al., 2009, 2011 a,b; Honda et al., 2010; Ichikawa et al., 2010). However, exactly which aspects of facial information are being processed in this face-selective region remains unanswered. In the present study, we explored the nature of facial representation in the temporal face-sensitive region found in the previous studies using the adaptation paradigm.

A growing number of recent adult fMRI studies have successfully employed the technique of fMRI-adaptation as a tool for examining the functional properties and the nature of representation processed in a particular neural population. fMRI-adaptation refers to the attenuation of fMRI signals to the repeated presentation of identical stimulus compared to the presentation of different stimuli (e.g., Grill-Spector et al., 2006). Many previous studies have reported that in the lateral occipital complex (LOC), which shows strong activity for objects, the repeated presentation of an identical object induced lower fMRI signals (Buckner et al., 1998; GrillSpector et al., 1998; Grill-Spector and Malach, 2001; Kourtzi and Kanwisher, 2001; Ewbank et al., 2005). Recently, specific regions of the face, the fusiform face area (FFA) and the superior temporal sulcus (STS; Kanwisher et al., 1997), also showed the same adaptation effect (Grill-Spector et al., 1999; Henson et al., 2000; Avidan et al., 2002; Henson and Rugg, 2003; Andrews and Ewbank, 2004). That is, the brain activity that occurs during the repeated presentation of an identical face is attenuated compared to brain activity during the presentation of different faces. The nature of the representation in these cortical areas was assessed by measuring the recovery from the adaptation when some stimulus properties were changed. If the fMRI signal remained adapted despite changes in the property of the stimulus, it indicates that the neuron is invariant to the property of the stimulus. On the other hand, if fMRI signals recovered from the adaptation, it means that the neuron is sensitive to the property of the stimulus. Using the technique of fMRI-adaptation and assessing the recovery from fMRI-adaptation, previous adult studies revealed that the representation of faces in the temporal area is invariant in translation and size (Grill-Spector et al., 1999; Andrews and Ewbank, 2004). We contemplated that if the technique of adaptation can be used in infants, it would provide a powerful tool for assessing the functional properties of the infant brain.

As a first step in establishing the neural adaptation effect in infants in the present study, we examined whether the adaptation effect occurs in infants' brain activity. Based on our previous NIRS studies (Otsuka et al., 2007; Carlsson et al., 2008; Grossmann et al., 2008; Nakato et al., 2009, 2011 a,b; Honda et al., 2010; Ichikawa et al., 2010), we focused on the processing of faces in the temporal regions of the infant brain. Using NIRS, we compared infants' brain activity in the bilateral temporal regions during the presentation of an identical face and the presentation of different faces. In Experiment 1, we aimed to establish the NIRS-adaptation paradigm in infants. Using frontal facial images as stimuli, we compared infants' hemodynamic responses to the presentation of five different facial images (the different-face condition) with their responses to the repeated presentation of an identical facial image (the same-face condition). We hypothesized that brain activity would be attenuated for the same-face condition compared to that of the different-face condition as a result of the adaptation effect. To further examine the nature of facial representation using the adaptation paradigm, we presented faces in varying points of view in Experiment 2. That is, we compared the hemodynamic responses to the presentation of the same face seen from the different viewpoint (the same-face condition) with that to the different faces seen from the different viewpoint (the different-face condition). We hypothesized that if faces are represented in a view-invariant manner, responses to the same-face condition would be attenuated compared to those to the different-face condition.

\section{EXPERIMENT 1 \\ MATERIALS AND METHODS \\ Participants}

The final sample of Experiment 1 consisted of 12 healthy infants aged 5-8 months ( 8 boys and 4 girls, mean age 179.4 days, $\mathrm{SD}=38.5$ days). Fifteen additional infants were excluded because of an insufficient number of available trials (less than three trials for either the same-face or the different-face condition), crying, failure to look at stimuli, or motion artifacts. One infant's data was excluded because the optimal condition for the measurement could not be achieved due to hair interference (less than 20 intact channels). All infants were full-term at birth and healthy at the time of testing. The infants were recruited through newspaper advertisements. This study was approved by the Ethical Committee of the National Institute for Physiological Sciences, and written informed consent was obtained from the parents of the infant participants. The experiments were conducted according to the Declaration of Helsinki.

\section{Stimuli and design}

The stimuli for the baseline period consisted of full-color photo images of five vegetables, which were the same as those used in our previous studies (Otsuka et al., 2007; Nakato et al., 2009, 2011 a,b; Honda et al., 2010; Kobayashi et al., 2012). The stimuli for the test period consisted of full-color photo images of five female faces in a frontal viewpoint. The sizes of the stimuli were approximately $17.5^{\circ} \times 21^{\circ}$ for the faces, and $16.8^{\circ} \times 16.8^{\circ}$ for the vegetables. The facial expression of five female faces was neutral.

There were two conditions for the test period: the same-face condition and the different-face condition (Figure 1A). The duration of each trial was fixed for $10 \mathrm{~s}$. In the different-face condition, five young female faces were shown in random order at a rate of $0.5 \mathrm{~Hz}$ within a trial. Each image flashed twice for $800 \mathrm{~ms}$, and a 200-ms inter-stimulus interval was filled by the presentation of a fixation point (a small red cross). In the same-face condition, one of the five faces was selected for each trial, and was shown repeatedly throughout the 10-s trial. The face in the same-face condition differed trial by trial without repeat until all five faces were presented. The order of presentation of the five faces was randomized for each infant. As in the different-face condition, each face flashed for a duration of $800 \mathrm{~ms}$ and a $200-\mathrm{ms}$ ISI was filled with the 

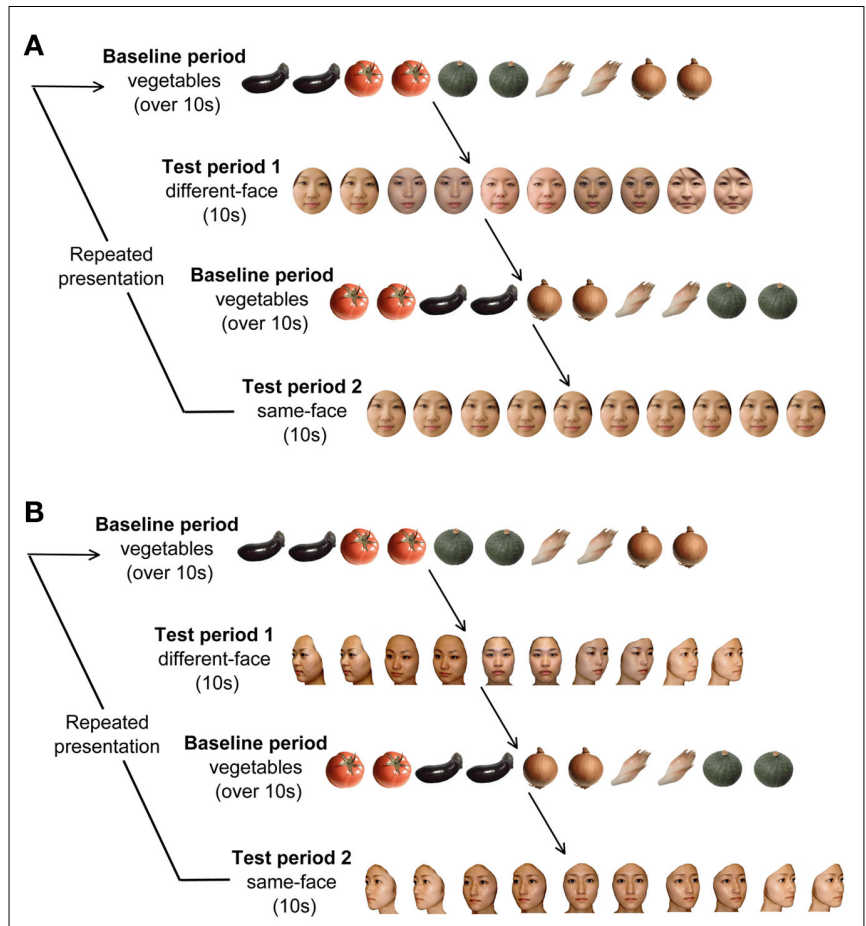

FIGURE 1 | (A) Experimental procedure in Experiment 1. In each trial, the baseline period consisted of stimuli of images of five vegetables, and its duration was at least $10 \mathrm{~s}$. The test period consisted of two conditions presenting frontal faces: the same-face condition and the different-face condition. The duration of the test period was fixed for $10 \mathrm{~s}$. The presentation order of test period 1 and 2 were changed alternately for each infant. (B) Experimental procedure in Experiment 2. The test period consisted of two conditions under the multiple viewpoint presentation: the same-face condition and the different-face condition.

fixation point. The same-face and different-face conditions were presented at alternating trials. The order of the presentation was counterbalanced across infants. The trials were repeated as long as infants were willing to look at stimulus display. The mean number of the trials presented for infants per condition was 10.8 trials for the different-face condition, and 10.3 trials for the same-face condition. The same-face and different-face condition followed the baseline period.

As in our previous studies (Otsuka et al., 2007; Nakato et al., 2009, 2011 a,b; Honda et al., 2010; Kobayashi et al., 2012), the five vegetables were shown in a random order at a rate of $0.5 \mathrm{~Hz}$ during the baseline period. As with the different-face condition, each vegetable's image flashed twice for a duration of $800 \mathrm{~ms}$, and a 200-ms ISI was filled with a fixation point (a small red cross). Each trial followed a baseline period of at least $10 \mathrm{~s}$. The duration of the baseline period was controlled by the experimenter. The results obtained from viewing objects were used as the baseline.

To draw and keep the attention of infants, both face stimuli and vegetables were accompanied by a beeping sound presented at $1 \mathrm{~Hz}$. Two different sounds were used for the face stimuli and the vegetables, and these sounds were used in both the same-face and different-face conditions. The relationship between the sounds and the visual stimuli was counterbalanced across infants.

\section{Apparatus}

Throughout the experiments, all stimuli were displayed on a 21-in color CRT monitor with a resolution of $1024 \times 768$ pixels controlled by a computer. The infant and CRT monitor were located inside an enclosure made of iron poles and covered with cloth. The distance between the infants and the monitor was approximately $40 \mathrm{~cm}$. There were two loudspeakers, one on either side of the CRT monitor. There was a CCD camera just below the monitor screen. Throughout the experiment, the infant's behavior was videotaped through this camera. The experimenter could observe the infant's behavior via a TV monitor connected to a pinhole camera.

\section{Procedure}

Each infant was tested while sitting on the experimenter's lap and facing a CRT monitor $40 \mathrm{~cm}$ away. The infants watched the stimuli passively while their brain activity was measured and they were allowed to watch the stimuli for as long as they were willing. The participants' behavior was videotaped during the experiment.

\section{Recording}

We used a HITACHI ETG-100 device system (Hitachi Medical, Chiba, Japan), which can record NIRS from 24 channels simultaneously, with 12 channels for the right temporal area, and 12 for the left. The instrument generated two wavelengths of NIR (780 and $830 \mathrm{~nm}$ ). The HITACHI ETG-100 measured the time-courses of the levels of oxyhemoglobin (oxy-Hb), deoxyhemoglobin (deoxy$\mathrm{Hb}$ ), and their sum (total-hemoglobin, total-Hb) at 24 channels with 0.1-s time resolution. Since we used newly developed NIRS sensor probes (Hitachi Medical, Infant probe $3 \times 3$ mode) for recording the infants, which have a lighter weight than previous probes and make softer contact with the skin, it was observed that most infants appeared to enjoy the experiments and were not reluctant to participate. We used a pair of probes, each containing nine optical fibers $(3 \times 3$ arrays $)$. Of the nine fibers, five were emitters, and four were detectors. The optical fibers of each probe were kept in place with a soft silicon holder. The distance between the emitters and detectors was set at $2 \mathrm{~cm}$ because each pair of adjacent emitting and detecting fibers were defined a single measurement channel, which allowed for the measurement of oxy- $\mathrm{Hb}$ and deoxy-Hb changes in 12 channels for each hemisphere.

In each hemisphere, the placement of the probes covered the temporal area centered at T5 and T6 according to the International 10-20 system (Jasper, 1958). This was a more posterior region than that of our previous study (Otsuka et al., 2007) and the same region as that of our recent studies (Nakato et al., 2009, 2011 a,b; Honda et al., 2010; Ichikawa et al., 2010; Kobayashi et al., 2012), since the posterior region of the temporal lobe is thought to be more important for face perception than the anterior and middle regions (Puce et al., 1996; Kanwisher et al., 1997; Halgren et al., 1999; Kanwisher and Yovel, 2006; see Figure 2).

When the probes were positioned, the experimenter checked to see if the fibers were touching each infant's scalp correctly. The Hitachi ETG-100 systems automatically detect whether the contact is adequate to measure the emerging photos for each channel. The channels were rejected from the analysis if adequate contact between the fibers and each infant's scalp could not be achieved because of hair interference. 


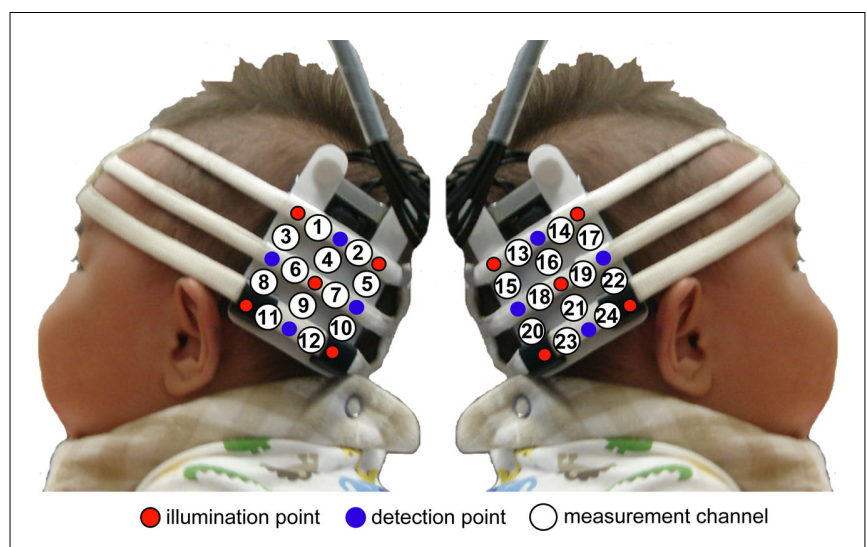

FIGURE 2 | Location of the probe and the measurement channels. The fibers were placed on the left and right temporal areas centering at the T5 and 6 of the International 10-20 system. The distance between the fibers was set at $2 \mathrm{~cm}$.

\section{Data analysis}

Throughout the experiment, the infants' behavior was recorded on videotape. We removed the trials from analysis if (1) the infants did not look at the test stimuli for at least the first $7 \mathrm{~s}$ of the 10s presentation or if they became fussy, (2) if the infants looked back at the face of the experimenter during the preceding baseline period, or (3) if the trials included movement artifacts which were detected by the analysis of sharp changes in the time series of the raw data of the NIRS.

On the basis of the wavelengths of the ETG-100 model (780 and $830 \mathrm{~nm})$, the estimations of oxy-Hb and total-Hb concentrations are more precise than those of the deoxy-Hb concentration (Pena et al., 2003; Otsuka et al., 2007). Since one of the advantages of NIRS over $\mathrm{fMRI}$ is its ability to measure the concentration of both oxy- and deoxy-Hb, the data of oxy-Hb, deoxy- $\mathrm{Hb}$, and total- $\mathrm{Hb}$ concentrations in the right and left temporal areas were used for the analysis in the present study.

The raw data of oxy-Hb, deoxy-Hb, and total-Hb from individual channels were digitally high-pass-filtered at $0.02 \mathrm{~Hz}$ to remove any longitudinal signal drift (Taga et al., 2003; Otsuka et al., 2007; Nakato et al., 2009, 2011 a,b; Honda et al., 2010; Kobayashi et al., 2012). Then the raw data of each channel were averaged across the trials within a subject in a time series of $0.1 \mathrm{~s}$ time resolutions from $2 \mathrm{~s}$ before the test trial onset to $10 \mathrm{~s}$ after the test trial offset.

From the time series of raw data of oxy-Hb, deoxy-Hb, and total-Hb, we calculated $Z$-scores at each time point to examine deviation of hemodynamic response to the presentation of faces from the baseline period where objects (vegetables) were shown. The $Z$-scores were calculated separately for oxy- $\mathrm{Hb}$, deoxy- $\mathrm{Hb}$, and total-Hb in the same-face and different-face condition for each channel within a subject. The $Z$-scores were calculated as the difference of the means of the baseline and test condition divided by the SD of the baseline using the following formula:

$d=\left(m_{\text {test }}-m_{\text {baseline }}\right) / s$

Accordingly, $m_{\text {test }}$ represents the averaged value of raw data during the test trials (different-face and same-face condition) and $m_{\text {baseline }}$ represents that of raw data during the vegetable baseline period. $s$ represents the SD of the baseline. In the present study, the "baseline" to calculate the $Z$-score means the mean value of $2 \mathrm{~s}$ immediately before the beginning of the each test condition within the baseline period which reflects the activation during the observation of vegetable images. Then the $Z$-scores obtained from 12 channels within each measurement area were averaged in order to increase the signal-to-noise ratio. Although the raw data of NIRS were originally relative values, and could not be averaged directly across subjects or channels, the normalized data such as the $Z$-scores could be averaged regardless of the unit (Schroeter et al., 2003; Matsuda and Hiraki, 2006; Shimada and Hiraki, 2006).

Consistent with previous studies using NIRS (Nakato et al., 2009, 2011a,b; Honda et al., 2010), we found that a response peak lag a few seconds behind stimulus onset (see Figure 3). Therefore, we performed statistical analyses against the mean $Z$-scores from 3 to $7 \mathrm{~s}$ after the face stimulus onset. A two-tailed one-sample $t$-test against a chance level of 0 (baseline) was conducted for the mean $Z$-score during the 3- to 7-s of the test trials in the left and right temporal areas. Furthermore, for all 24 channels, each channels' activation was tested by a two-tailed one-sample $t$-test against the baseline. To eliminate the risk of a Type I error, we performed the corrections using the false discovery rate (FDR; Singh and Dan, 2006).

Finally, a channels-of-interest (COI) was conducted for the channels which showed a significant increase in the concentration of oxy-Hb in the different-face condition compared to the baseline period (presentation of objects). We conducted a repeatedmeasure ANOVA with two within-subject factors, condition and channels on the COI region.

\section{RESULTS}

We obtained hemodynamic responses from 12 infants who looked at the stimuli for more than three trials in both the same-face and different-face conditions. The mean number of trials was 5.33 for the same-face condition, and 5.41 for the different-face condition. The mean number of channels was 11.88 for both the right and left temporal areas.

Figure 3 shows the time-course of the average change of the oxy-, deoxy-, and total-Hb concentrations while the infants looked at either the same-face or different-face condition. Zero on the horizontal axis represents the beginning of the test period and 10 on the horizontal axis represents the end of the test period. The graphs in the left column show data from the left temporal area (1-12 channels), and those in the right column show data from the right temporal area (13-24 channels). The black line in the graphs represents the mean $Z$-score of the different-face condition, and the gray line represents the mean $Z$-score of the same-face condition. In both conditions, the hemodynamic changes of deoxy- $\mathrm{Hb}$ and total- $\mathrm{Hb}$ were smaller than those of oxy-Hb. The concentration of oxy-Hb in both the left and right temporal areas showed greater increase during the different-face condition than during the same-face condition. These increases in the different-face condition occurred at about $3 \mathrm{~s}$ after the onset of the stimuli and the decrease occurred at about $7 \mathrm{~s}$.

Figure 4 shows the mean $Z$-score from 3 to $7 \mathrm{~s}$ of the trial in the left and right temporal areas. A repeated-measure ANOVA 

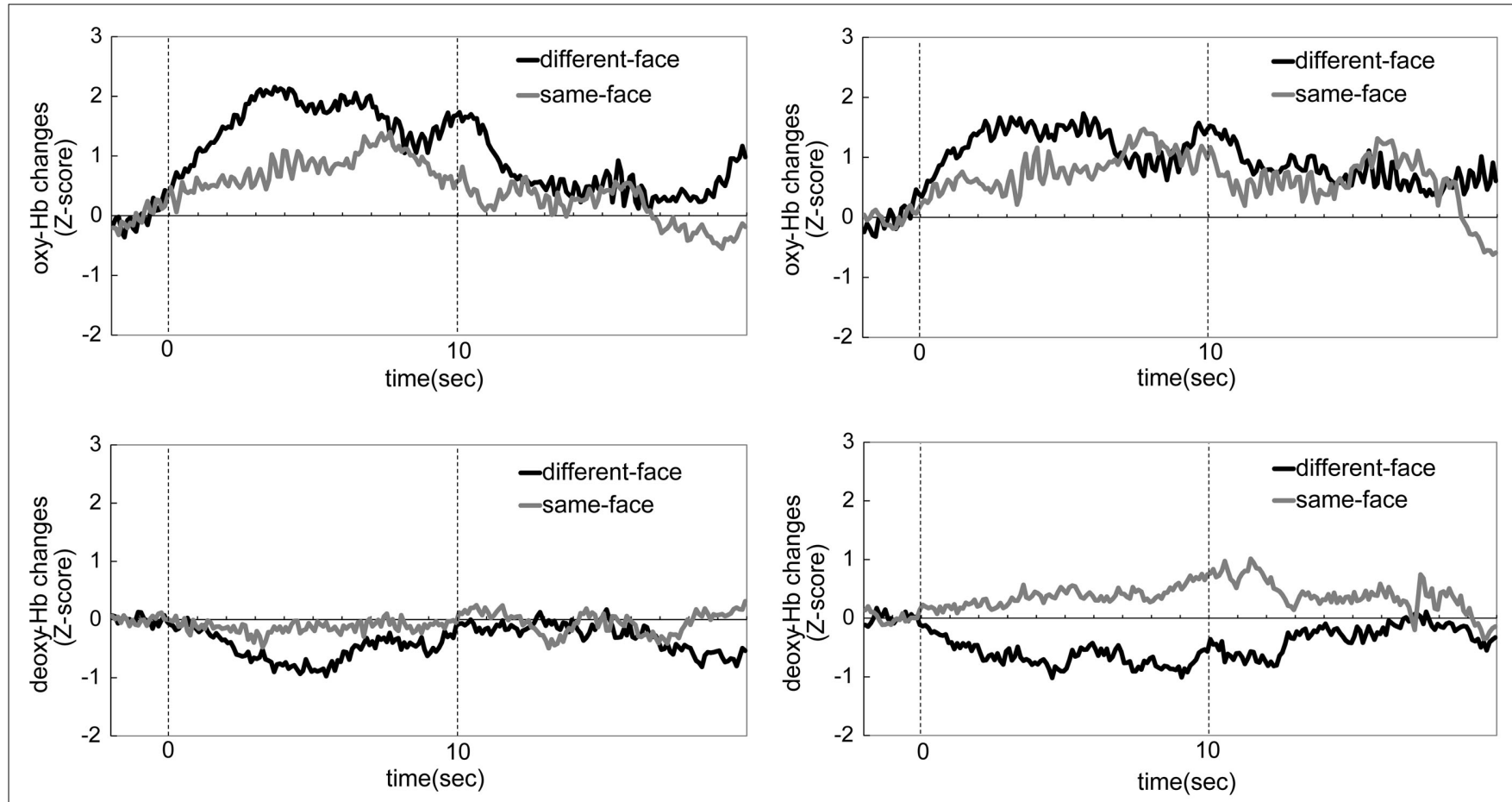

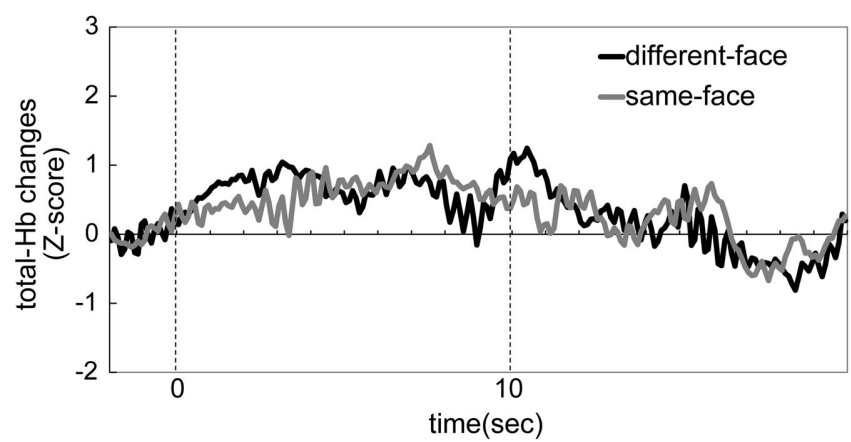

Left temporal area

FIGURE 3 | The time-course of the average change in oxyhemoglobin (oxy-Hb), deoxyhemoglobin (deoxy-Hb), and total-hemoglobin (total-Hb) concentrations during the same-face condition and the different-face condition in Experiment 1. The left column shows data from the left temporal area, and the right column shows those from the right temporal

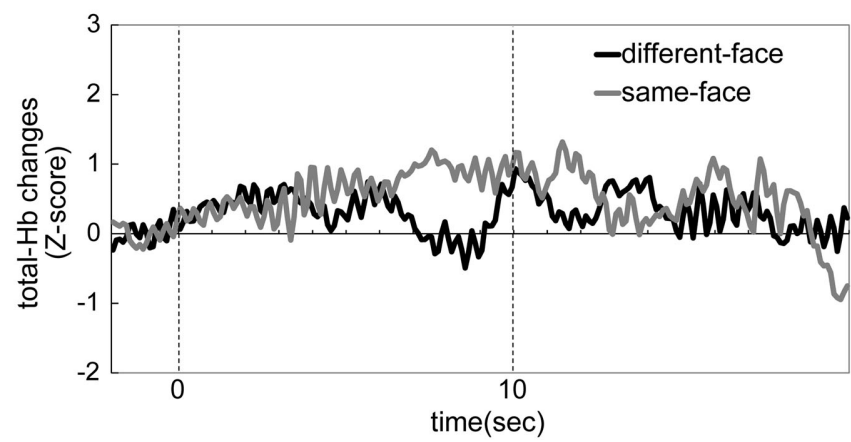

Right temporal area

area. The black line in the graph represents the mean $Z$-score of the different-face condition, and gray line represents the mean $Z$-score of the same-face condition. Zero on the horizontal axis represents the beginning of the test period and 10 on the horizontal axis represents the end of the test period. with condition (same-face vs. different-face) and measurement area (right vs. left) as the within-subject factors was separately performed on the data of oxy-, deoxy-, and total-Hb. This analysis revealed a marginally significant effect of condition for oxy- $\mathrm{Hb}$ $[F(1,11)=3.43, p=0.091]$. The effect of measurement area and interaction was not significant. In addition, no significant effect or interaction was found for deoxy- $\mathrm{Hb}$ and total-Hb. To examine the possibility that there was differential activity for the observation of faces compared to the baseline period where vegetables were shown, we performed a two-tailed one-sample $t$-test on the $Z$-scores against a chance level of 0 (baseline) for each condition and hemisphere separately. The analysis revealed that the concentration of oxy-Hb increased significantly in both the left and right temporal area during the different-face condition [left: $t(11)=3.19, p<0.01$; right: $t(11)=3.64, p<0.01]$. The concentration of deoxy-Hb decreased significantly in the right temporal area during the different-face condition $[t(11)=-2.25, p<0.05]$. On the other hand, no significant change in the concentration of oxy-Hb or deoxy-Hb was found during the same-face condition $(p>0.10)$. In addition, no significant change was found for the concentration of total-Hb in either condition.

Table 1 illustrates the channels which showed a significant activation from 3 to $7 \mathrm{~s}$ after the stimulus onset compared to the baseline in the different-face condition. The $Z$-score in the oxy$\mathrm{Hb}$ was maximal around the T5 position in the left temporal area and around the T6 position in the right temporal area. During 


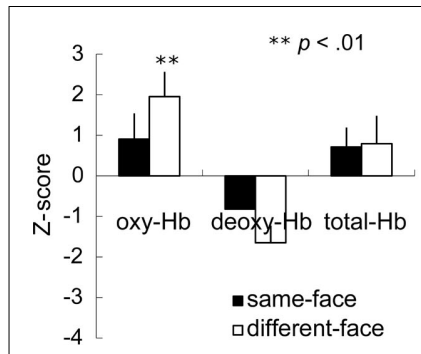

Left temporal area

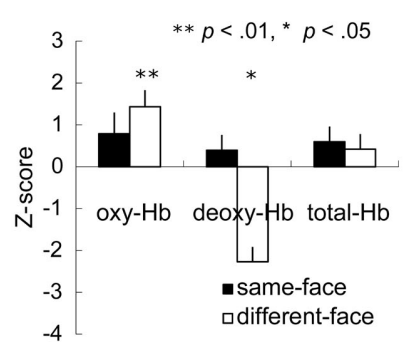

Right temporal area
FIGURE 4 | Mean Z-score from 3 to $7 \mathrm{~s}$ of the trial in the bilateral temporal area in the Experiment 1. The vertical line in the graphs represents $1 \mathrm{SE}$. In the different-face condition (white bar), the concentration of oxy- $\mathrm{Hb}$ in both the right and left temporal areas increased significantly compared to the chance level of $0\left({ }^{* *} p<0.01,{ }^{*} p<0.05\right)$.

Table 1 | Channels activated in the different-face condition (Experiment 1) compared to the baseline.

\begin{tabular}{lll}
\hline & \multicolumn{2}{c}{ Oxy-Hb } \\
\cline { 3 - 3 } & ch. & Z-score \\
\hline \multirow{2}{*}{ Left } & 9 & $2.83^{*}$ \\
& 10 & $3.37^{*}$ \\
Right & 18 & $4.03^{*}$ \\
& 23 & $3.09^{*}$ \\
& 24 & $3.28^{*}$
\end{tabular}

ch., channel.

${ }^{*} p<0.05$ : two-tailed one-sample t-test vs. chance level of 0 . The significant $p$-value was determined by the false discovery rate (FDR).

the presentation of the same-face condition, no channel showed a significant change in activation. Furthermore, no significant change in individual channel was found for the concentration of deoxy-Hb and total-Hb either in the same-face or the different-face condition.

The five channels (ch. 9, 10, 18, 23, and 24) that showed significantly higher activation for face than for the object baseline in the different-face condition (Table 1) were selected as COI. Figure 5 represents the $Z$-scores of the five channels selected as $\mathrm{COI}$ in the same-face condition and the different-face condition, respectively. All of these channels showed higher $Z$-scores in the different-face condition than those in the same-face condition for oxy-Hb. A repeated-measure ANOVA with condition (same-face vs. different-face) and channels (ch. 9, 10, 18, 23, 24) as the withinsubject factors was performed on the data of oxy-, deoxy-, and total-Hb. This analysis revealed a significant effect of condition for oxy-Hb $[F(1,9)=14.42, p<0.01]$. The effect of channels and interaction were not significant. In addition, no significant effect or interaction was found for deoxy-Hb and total-Hb.

Although only the marginal significant effect of condition was found as the results of ANOVA on the averaged $Z$-score of 12 channels for each hemisphere, the significant effect of condition was shown in the five channels around T5 and T6 position. These

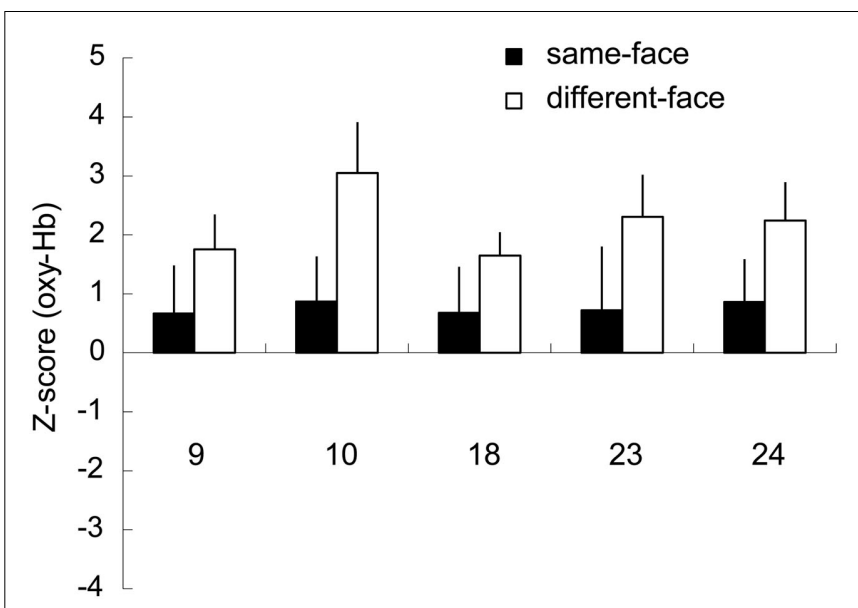

channel

FIGURE 5 | Mean Z-scores of the five channels (ch. 9, 10, 18, 23, 24) selected as $\mathrm{COI}$ in the same-face and the different-face conditions, respectively. The vertical line in each graph represents $1 \mathrm{SE}$. All of these channels showed the higher Z-scores in the different-face condition compared to those in the same-face condition for oxy- $\mathrm{Hb}$. The main effect of condition was significant with 2 (condition) $\times 5$ (channel) ANOVA $(p<0.01)$.

results suggest that the adaptation to the face occur within the relatively specific region of temporal area in infants.

\section{EXPERIMENT 2}

In Experiment 1, we found a significantly lower hemodynamic response during the presentation of an identical face than during the presentation of different faces, showing the adaptation effect in infant hemodynamic responses for faces. In Experiment 2 , we examined whether this adaptation would occur when multiple views of a face were presented. We compared hemodynamic responses in the same-face condition and in the different-face condition as in Experiment 1, however, faces in both conditions changed viewpoint within each trial. An attenuated response in the same-face condition compared to that in the different-face condition suggests a viewpoint-invariant representation of faces.

In our previous study, we found some developmental changes in the processing of the faces in different views within the age range we examined in Experiment 1. That is, we found that the presentation of frontal face images induced increased response compared to the responses to objects in both 5- and 8-month-olds, while only 8-month-olds showed such response to facial images in the profile view (Nakato et al., 2009). Based on the findings of Nakato et al. (2009), we divided infants into two age groups (5-6, 78 months) and compared NIRS responses to examine the possible developmental change in the processing of faces in multiple views.

\section{MATERIALS AND METHODS}

The methods in Experiment 2 were the same as those in Experiment 1 except for the following.

\section{Participants}

The final sample of Experiment 2 consisted of 24 healthy infants, twelve 5 - to 6 -month-olds ( 7 boys and 5 girls, mean age 169.4 days, 
$\mathrm{SD}=18.7$ days) and twelve 7 - to 8-month-olds ( 5 boys and 7 girls, mean age 226.3 days, $S D=16.2$ days). Six additional infants were excluded based on the same criteria as Experiment 1.

\section{Stimuli and design}

The stimulus set used for the experiment consisted of full-color photo images of five female faces viewed from five viewpoints ranging from the left three-quarter view to the right three-quarter view in increments of $22.5^{\circ}$ (Figure 1B). In each trial, infants were shown a sequence of five facial images that gradually change in viewpoint from the left- to right three-quarter view or vice versa. The left and right orientation of the first image in each trial was determined randomly. In the different-face condition, each of the five images differed in identity as well as in viewpoint, while they differed only in viewpoint in the same-face condition. Since the 200-ms interval in between the facial images was filled by the presentation of a fixation point, the changes in the viewpoint of faces did not induce the perception of facial movement.

In 5-6 months, the mean number of the trials presented for infants was 8.6 trials for the different-face condition, and 8.2 trials for the same-face condition. In 7-8 months, the mean number of the trials presented for infants was 8.8 trials for the different-face condition, and 8.6 trials for the same-face condition.

\section{Recordings}

We used a newly developed machine, the HITACHI ETG-4000 device system (Hitachi Medical, Chiba, Japan), which can record NIRS from 24 channels simultaneously, with 12 channels for the right temporal area, and 12 for the left. The instrument generates two wavelengths of NIR (695 and $830 \mathrm{~nm}$ ). The wavelength of NIR differs between ETG-100 and ETG-4000: ETG-100 = 780, $830 \mathrm{~nm}$ and ETG-4000 $=695,830 \mathrm{~nm}$. Long wavelength $(830 \mathrm{~nm})$ irradiated by two systems contributes to detection of changes in the concentration of oxy-Hb, whereas short wavelength (780 or $695 \mathrm{~nm}$ ) contributes to detection of changes in the concentration of deoxy-Hb (Matcher et al., 1995). In the current study, we focused on the changes in the concentration of oxy-Hb in interpretation of data. As there is no change in the high wavelength of NIR between ETG-100 and ETG-4000 for the measurement of oxy-Hb, we believe that the differences in the specifications between the two systems had little affected on our results.

\section{RESULTS}

We obtained hemodynamic responses from 12 5- to 6-monthold and 12 7- to 8-month-old infants who looked at the stimuli for more than three trials in both the same-face and different-face conditions. For the 5- to 6-month-olds, the mean number of trials was 4.33 for the same-face condition, and 4.75 for the different-face condition. For the 7- to 8-month-olds, the mean number of trials was 4.91 for the same-face condition and 4.33 for the different-face condition. The mean number of channels was 12.0 for the left temporal area, and 11.9 for the right temporal area in the younger infant group. The mean number of channels was 11.9 for the left temporal area, and 12.0 for the right temporal area in the older infant group.

Figure 6 shows the time-course of the average change of the oxy-, deoxy-, and total-Hb concentrations in the 5- to 6-monthold and the 7-to 8-month-old groups when viewing the same-face

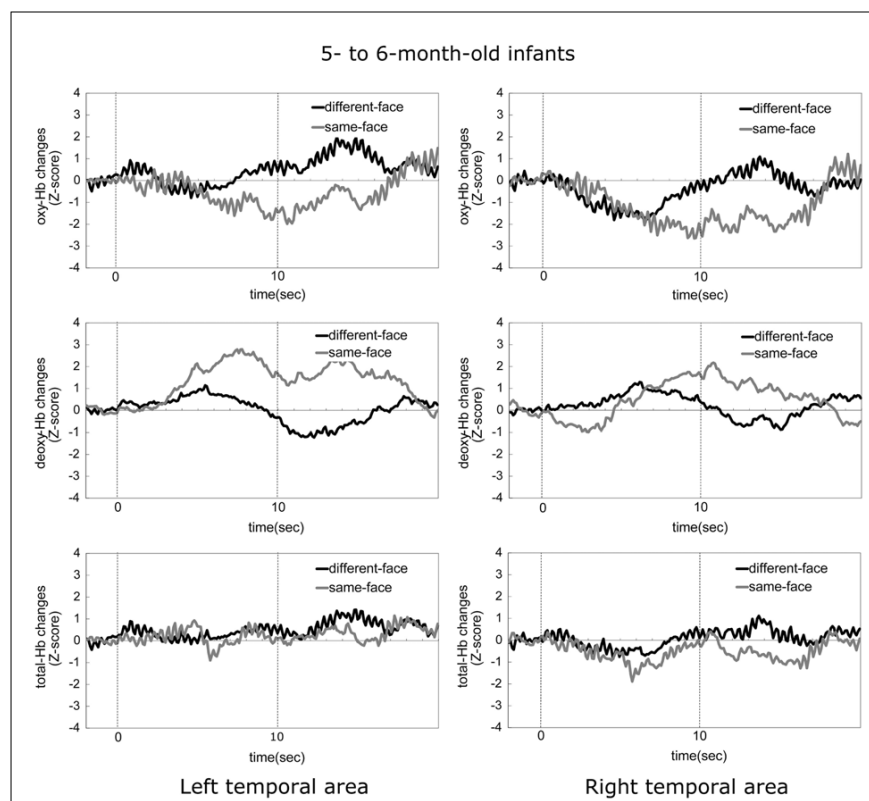

FIGURE 6 | The time-courses of the average change in oxy-, deoxy-, and total-Hb concentrations during the same-face and different-face condition under the multiple viewpoint presentation in 5- to 6-month-old infants and 7- to 8-month-old infants in Experiment 2. In both age groups, the two graphs in the top part indicate the data for oxy-Hb, the two graphs in

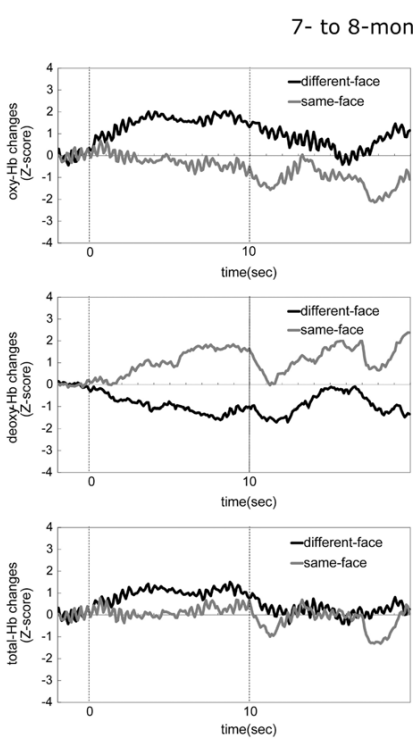

Left temporal area
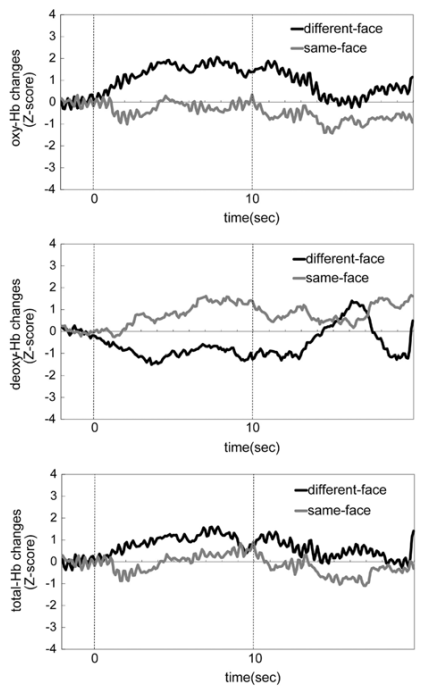

Right temporal area the middle part for deoxy-Hb, and the two graphs in the bottom part for total- $\mathrm{Hb}$. The data for the left temporal area are shown in the left column and those for the right temporal area are shown in the right column. In each graph, the black line represents the mean Z-score of the different-face condition and the gray line represents that of the same-face condition. 
and different-face conditions. Zero on the horizontal axis represents the beginning of the test period and 10 represents the end of the test period. The black line in the graph represents the mean $Z$-score of the different-face condition and the gray line represents the mean $Z$-score of the same-face condition.

At 5-6 months of age, the concentration of deoxy-Hb in both the right and left temporal areas increased only in the same-face condition. Oxy- and total-Hb showed a similar tendency between the different-face and same-face conditions. That is, compared to the baseline, the increases in the concentration of oxy- and total-Hb were not shown in both temporal areas.

At 7-8 months of age, the concentration of oxy- and total$\mathrm{Hb}$ in bilateral temporal areas showed a greater increase in the different-face condition than in the same-face condition. These increases in the different-face condition occurred at about $4 \mathrm{~s}$ after the onset of the face stimuli, peaking around 5-7 s. In line with oxy- and total-Hb changes, the concentration of deoxy- $\mathrm{Hb}$ showed a greater decrease in the different-face condition than in the same-face condition in both the left and right temporal regions.

Figure 7 shows the mean $Z$-score from 3 to $7 \mathrm{~s}$ after the onset of facial images across the 12 channels in the left and right temporal areas. A repeated-measure ANOVA with age (5-6 vs. 7-8 months) as a between-subject factor, and condition (same-face vs. differentface) and measurement area (right vs. left) as the within-subject factors, was separately performed on the data of oxy-, deoxy-, and total-Hb. This analysis revealed a significant main effect of age for oxy- $\mathrm{Hb}[F(1,22)=5.73, p<0.05]$ and total-Hb $[F(1$, $22)=4.47, p<0.05]$. In addition, there was a marginally significant interaction between age and condition for oxy-Hb $[F(1$, $22)=3.65, p=0.06]$, suggesting a greater difference between the two test conditions for 7- to 8-month-olds than for 5- to 6-montholds. No other effect or interaction reached statistical significance $(p>0.10)$.

To examine the possibility that there was different activity for the observation of faces compared to the baseline period where vegetables were shown, we performed a two-tailed one-sample $t$-test against a chance level of 0 (baseline) on the $Z$-scores of oxy-, deoxy-, and total-Hb for each age group separately. In 5- to 6-month-old group, the concentration of oxy-Hb decreased significantly in the right temporal area during the presentation of the different-face condition $[t(11)=-6.14, p<0.01]$, while the concentration of deoxy-Hb increased significantly in the left temporal area during the same-face, condition $[t(11)=2.55, p<0.05]$. No significant change in the total-Hb was observed in either of the conditions $(p>0.10)$. In 7 - to 8 -month-old group, there was a significant increase in the concentrations of oxy-Hb [left: $t(11)=2.89, p<0.05$, right: $t(11)=4.32, p<0.01]$ and total-Hb [left: $t(11)=2.44, p<0.05$, right: $t(11)=2.27, p<0.05$ ] during the different-face condition in both temporal regions. In contrast, no such increase in the concentration of oxy- $\mathrm{Hb}$ and total-Hb was found in the same-face condition $(p>0.10)$. No significant change was found for the concentration of deoxy-Hb in either condition.

Table 2 illustrates the channels that showed significant activation from 3 to $7 \mathrm{~s}$ after the stimulus onset in 7- to 8-month-olds during the different-face condition compared to the baseline. The $Z$-score in the oxy-Hb was maximal around the T6 position in

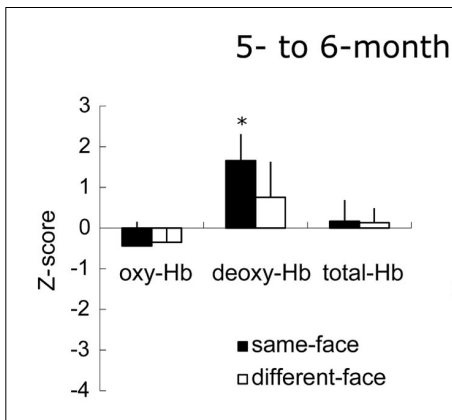

Left temporal area

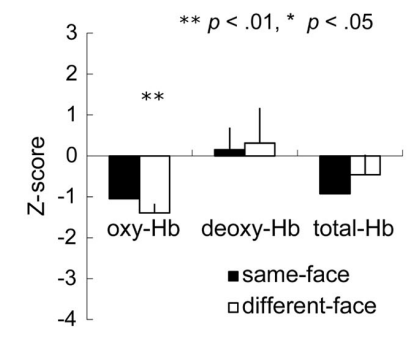

Right temporal area

\section{7- to 8-month-old infants}

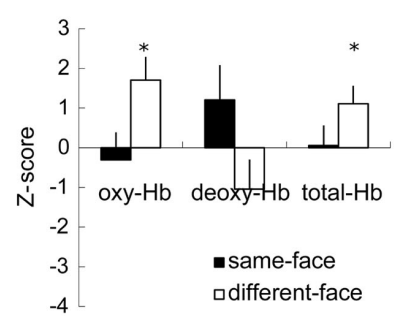

Left temporal area

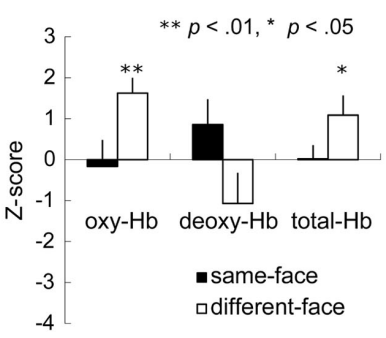

Right temporal area
FIGURE 7 | Mean Z-score from 3 to $7 \mathrm{~s}$ after stimulus onset in the bilateral temporal area in Experiment 2. The vertical line in the graphs represents 1 SE. At 5-6 months of age, the concentration of deoxy-Hb significantly increased during the same-face condition under the different viewpoint presentation only in the left temporal area. Furthermore, the decrease in oxy-Hb concentration was significant in the right temporal area while infants looked at the different-face condition. At 7-8months, in the different-face condition (white bar), the concentration of oxy-Hb and total-Hb in both the right and left temporal areas increased significantly compared to the chance level of $0\left({ }^{* *} p<0.01,{ }^{*} p<0.05\right)$.

the right temporal area. The location of channels with significant activation is in close proximity to what was found in Experiment 1. In contrast to the different-face condition, no channels showed a significant change in hemodynamic responses in the same-face condition. No further channels with significance were found for the concentration of deoxy-Hb and total-Hb. In addition, no significant channels were identified for the 5- to 6-month-old group in either condition.

We further analyzed the data from the 7-to 8-month-old group based on COI. The six channels (ch. 9, 18, 20, 22, 23, and 24) that showed a significantly higher response for the different-face condition than for the object baseline (Table 2) were selected as COI. Figure 8 represents the $Z$-score in oxy-Hb of the six channels selected as COI in the same-face and different-face condition, respectively. All of these channels showed a higher $Z$-score in the different-face condition than in the same-face condition. A repeated-measure ANOVA with two factors, condition (same-face vs. different-face) and channels (ch. 9, 18, 20, 22, 23, and 24) as the within-subject factors, was performed on the data of oxyHb. This analysis revealed a significant effect of condition $[F(1$, $11)=11.95, p<0.01]$. The effect of channels and interaction were not significant. 
Table 2 | Channels activated in the different-face condition (Experiment 2) compared to the baseline on 7- to 8-month-old infants.

\begin{tabular}{llllll}
\hline & \multicolumn{2}{c}{ Oxy-Hb } & & \multicolumn{2}{c}{ Total-Hb } \\
\cline { 2 - 3 } & ch. & Z-score & & ch. & Z-score \\
\hline Left & 9 & $1.77^{*}$ & 7 & $1.67^{*}$ \\
Right & 18 & $2.66^{*}$ & & \\
& 20 & $1.73^{*}$ & & \\
& 22 & $2.04^{*}$ & & \\
& 23 & $1.04^{*}$ & & \\
& 24 & $2.43^{*}$ & & \\
\hline
\end{tabular}

ch., channel.

${ }^{*} p<0.05$ : two-tailed one-sample $t$-test vs. chance level of 0 . The significant $p$-value was determined by the false discovery rate (FDR).

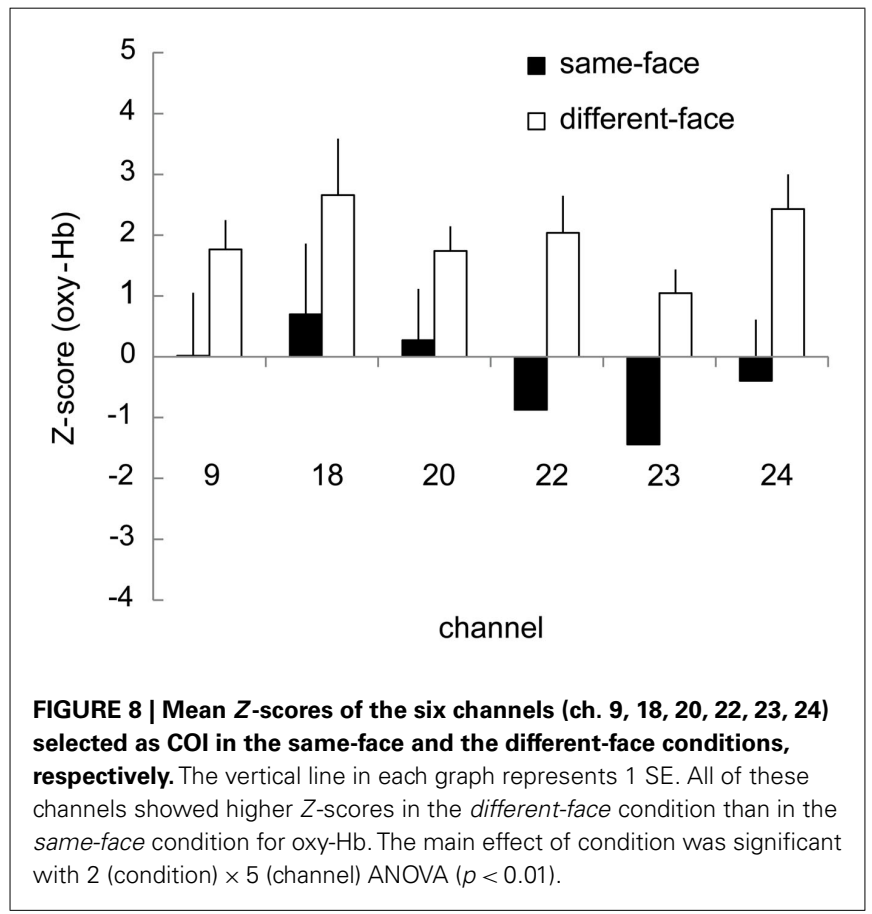

\section{DISCUSSION}

In the present study, we used the neural adaptation paradigm to examine the nature of facial representation in infants aged 58 months of age. To establish the neural adaptation effect in infants as measured by NIRS, we compared hemodynamic responses between the repeated presentation of the same face (the sameface condition) and the presentation of five different faces (the different-face condition) in Experiment 1. We hypothesized that neural adaptation would lead to a lower hemodynamic response to the repeated presentation of an identical face than to that of various faces. As a result, we found that channels in the bilateral temporal region around the $\mathrm{T} 5$ and $\mathrm{T} 6$ areas, which are more responsive to faces than to objects, showed a significant difference between the same-face condition and the different-face condition. That is, the concentration of oxy-Hb in these channels was significantly higher during the different-face condition than during the same-face condition. In Experiment 2, we measured the hemodynamic responses in the same-face and the differentface conditions again, but faces in both conditions underwent changes in viewpoint. We found that at 7-8 months of age, but not at 5-6 months of age, infants showed differential responses between the same-face condition and the different-face condition under changes in viewpoint.

Our findings in Experiment 1 demonstrate the neural adaptation effect for the repeated presentation of a face based on the hemodynamic responses in infants' brain as measured by NIRS (NIRS-adaptation). The attenuated hemodynamic response to the repeated presentation of an identical face compared to those of different faces is consistent with findings from previous fMRI studies in adults showing the neural adaptation effect in face recognition (e.g., Grill-Spector et al., 1999; Andrews and Ewbank, 2004; Ewbank and Andrews, 2008). The attenuated hemodynamic responses induced by the repeated presentation of an identical face suggest that the infants' temporal region around T5 and T6 plays an important role in the discrimination of faces and/or recognizing faces. This is consistent with our previous findings using NIRS that the posterior regions around $\mathrm{T} 5$ and $\mathrm{T} 6$ are particularly important for infants' face recognition (Nakato et al., 2009, 2011a,b; Honda et al., 2010; Ichikawa et al., 2010). In the right temporal area, the channels around the T6 position, especially the inferior channels, showed increases in the concentration of oxy-Hb. The channels activated in our study were in close proximity to those in Nakato et al. (2009) and Nakato et al. (2011a,b). In the left temporal area, the region activated in the present study was adjacent to the channels activated by the presentation of canonical faces as observed by Honda et al. (2010). Our results are also consistent with previous neuroimaging studies of adults reporting the importance of the $\mathrm{T} 5$ and $\mathrm{T} 6$ regions of the temporal lobe for face perception (Puce et al., 1996; Kanwisher et al., 1997; Halgren et al., 1999; Kanwisher and Yovel, 2006).

Our results from Experiment 2 suggest that face is represented in a view-invariant manner in the temporal region of infant brains at $7-8$ months of age. The results of the adaptation effect for the same face seen from various viewpoints would rule out the possibility that greater brain responses to the different faces rather than an identical face in Experiment 1 reflect the discrimination of images in general based on the low-level image properties. Our findings are consistent with a recent study of adults showing that the temporal region including STS shows neural adaptation to high-level properties rather than low-level stimulus properties (Zevin et al., 2010). This pattern of activation suggests that the region is involved in representation of faces rather than general image discrimination processing at $7-8$ months of age.

While we found no developmental trend in the adaptation effect in infants aged 5-8 months in Experiment 1, we found a developmental change in Experiment 2. In contrast to 7- to 8month-olds, 5- to 6-month-olds did not show the adaptation effect in Experiment 2 when shown the faces in various viewpoints. This developmental change is consistent with our previous findings from NIRS study and other previous studies. In the previous NIRS study, we found that the temporal area at 8 months of age, but not at 5 months of age, responds to faces differently than to objects not only when the frontal faces are shown, but 
also when profile faces are shown (Nakato et al., 2009). By using ERPs, Gliga and Dehaene-Lambertz (2007) reported that they did not find the evidence of view-invariant facial representation in 4 months of age. Consistent with these neuroimaging studies in infants, most behavioral studies have reported the ability to recognize the faces in various viewpoints develops over 7 months of age (Fagan, 1976; Cohen and Strauss, 1979; Rose et al., 2002; Nakato et al., 2010). These previous neuroimaging and behavioral studies suggest that neural basis of processing faces shown in various viewpoint develop later in infancy.

Though we did not find evidence of view-invariant representation of faces in 5- to 6-month-old infants, our results do not necessarily show that they lack the ability to recognize face across different viewpoints. Several studies have demonstrated the ability to recognize faces across the different viewpoints even in infants younger than 6 months of age (Turati et al., 2004, 2008; Bulf and Turati, 2010). One possible explanation for the differential findings would be that young infants require a longer period to develop a facial representation which is invariant across views. In the current study, infants were required to recognize unfamiliar faces that were shown spontaneously in various viewpoints without any prior learning period. By contrast, the studies showing recognition across viewpoints in young infants generally used an infant-controlled habituation procedure in which infants were given considerable time to view the faces before the recognition test was conducted. Taken together, our results suggest that the ability to represent the face across viewpoints becomes more robust and efficient between 5 and 8 months of age.

Interestingly, we found that more channels in the right temporal area of 7- to 8-month-olds were activated by the presentation of faces shown under multiple viewpoints (Experiment 2) than those shown only in the frontal view (Experiment 1). Consistent with our results, Nakato et al. (2009) reported that the presentation of a profile face led to more channels being activated than the presentation of a frontal face in 8-month-old infants. In previous fMRI studies, it was shown that facial identity is represented in a view-invariant manner in medial fusiform gyrus adjacent to FFA (Pourtois et al., 2005a,b, 2009) but not in face-sensitive FFA (Grill-Spector et al., 1999; Andrews and Ewbank, 2004; Pourtois et al., 2005a; Fang et al., 2006; Ewbank and Andrews, 2008). Since analysis in most of the fMRI studies with adults was restricted to ROI, which was limited to functionally defined face-selective areas such as FFA and OFA (Grill-Spector et al., 1999; Andrews and Ewbank, 2004; Pourtois et al., 2005a; Fang et al., 2006; Ewbank and Andrews, 2008), it is unclear from these studies whether adults also show differences between the processing of faces seen from various viewpoints and faces seen from a single viewpoint. Based on the findings that responses in face-sensitive areas such as FFA showed limited generalizability of identity codes to image transformations such as viewpoint change, however, a recent fMRI study by Natu et al. (2010) has examined the possibility that the broader area in the ventral temporal cortex is involved in the processing of

\section{REFERENCES}

Andrews, T. J., and Ewbank, M. P. (2004). Distinct representations for facial identity and changeable aspects of faces in the human temporal lobe. Neuroimage 23, 905-913. T., Zohary, E., and Malach, R. (2002). Analysis of the
Avidan, G., Hasson, U., Hendler,

facial identity. In order to examine neural codes for facial identity without restricting the analysis to ROI, Natu et al. (2010) applied a pattern-based classification analysis to the task of discriminating faces by identity across a broader area of the cortex. They found that the broad area of the ventral temporal cortex, including fusiform gyrus and lateral occipital complex (LOC), was required for the neural discrimination of facial identity under changing viewpoints. Consistent with Natu et al. (2010), our results suggest that the processing of faces seen from multiple viewpoints requires an involvement of the broader region of the temporal area than that of faces seen from a single frontal viewpoint.

In the current study, we applied the neural adaptation paradigm same as adult fMRI studies and showed the neural adaptation effect in infancy. One of the problems in interpreting the results when using the same paradigm or task between NIRS and fMRI recordings is the comparability of signals detected by NIRS and that detected by fMRI. Previous studies conducting simultaneously measurement of NIRS and fMRI revealed that there is a strong correlation between signals measured with NIRS and fMRI (Toronov et al., 2001, 2007; Strangman et al., 2002). In the recent study, Cui et al. (2011) reported such correlation between NIRS and fMRI signals even for cognitive task. These results suggest that temporal attenuation in the NIRS signals induced by the repeated presentation of identical face shown in this study could be interpreted as the similar temporal attenuation reported by fMRI-adaptation studies.

The current study, applying the neural adaptation paradigm, used NIRS to explore the neural codes of the face in infants. We obtained the first evidence of the adaptation effect for face in the temporal area of the infant brain. We further found that the adaptation effect in these areas at 7-8 months of age was not sensitive to changes in viewpoint, suggesting a view-invariant representation of faces. Although we focused on the adaptation effect in facial processing, the ubiquitous nature of the adaptation effect suggests that this technique can be applicable to examine various kinds of perceptual and cognitive processes. As with the adult brain, the fNIRS-adaptation paradigm would be a strong tool for examining the functional properties of infants' developing brains.

\section{ACKNOWLEDGMENTS}

This research was supported by PRESTO (Japan Science and Technology Agency), a Grant-in-Aid for Scientific Research from JSPS (18300090, 21243041), a Grant-in-Aid for Scientific Research by JSPS Research Fellowships for Young Scientists (22 2637), and a Grant-in-Aid for Scientific Research on Innovative Areas, "Face perception and recognition" from the Ministry of Education, Culture, Sports, Science, and Technology KAKENHI (20119002). We are grateful to Prof. Norihiro Sadato, National Institute for Physiological Sciences, for his technical assistance. We also thank Yuko Hibi, Hiroko Ichikawa, Aki Tsuruhara, Midori Takashima, Jiale Yang, Yuka Yamazaki, and Wakayo Yamashita for their help in data collection.

neuronal selectivity underlying low fMR signals. Curr. Biol. 25, 964-972.

Baird, A. A., Kagan, J., Gaudette, T., Walz, T. A., Hershlag, N., and
Boas, D. A. (2002). Frontal lobe activation during object permanence: data from near-infrared spectroscopy. Neuroimage 16, 1120-1126. 
Bartocci, M., Winberg, J., Ruggiero, C., Bergqvist, L. L., Serra, G., and Lagercrantz, H. (2000). Activation of olfactory cortex in newborn infants after odor stimulation: a functional near-infrared spectroscopy study. Pediatr. Res. 48, 18-23.

Bortfeld, H., Wruck, E., and Boas, D. A. (2007). Assessing infants' cortical response to speech using nearinfrared spectroscopy. Neuroimage $34,407-415$.

Buckner, R. L., Goodman, J., Burock, M., Rotte, M., and Koutstaal, W. (1998). Functional-anatomic correlates of object priming in human revealed by rapid presentation event-related fMRI. Neuron 20, 285-296.

Bulf, H., and Turati, C. (2010). The role of rigid motion in newborn's face recognition. Vis. Cogn. 80, 504-512.

Carlsson, J., Lagercrantz, H., Olson, L., Printz, G., and Bartocci, M. (2008). Activation of right fronto-temporal cortex during maternal facial recognition in young infants. Acta Paediatr. 97, 1221-1225.

Cohen, L. B., and Strauss, M. S. (1979). Concept acquisition in the human infant. Child Dev. 50, 419-424.

Cui, X., Bray, S., Bryant, D. M., Glover, G. H., and Reiss, A. L. (2011). A quantitative comparison of NIRS and fMRI across multiple cognitive tasks. Neuroimage 54, 2808-2821.

Ewbank, M. P., and Andrews, T. J. (2008). Differential sensitivity for viewpoint between familiar and unfamiliar faces in human visual cortex. Neuroimage 40, 1857-1870.

Ewbank, M. P., Schluppeck, D., and Andrews, T. J. (2005). fMRadaptation reveals a distributed representation of inanimate objects and places in human visual cortex. Neuroimage 28, 268-279.

Fagan, J. F. (1976). Infants' recognition of invariant features of faces. Child Dev. 47, 627-638.

Fang, F., Murray, S. O., and He, S. (2006). Duration-dependent fMRI adaptation and distributed viewer-centered face representation in human visual cortex. Cereb. Cortex 17, 1402-1411.

Gliga, T., and Dehaene-Lambertz, G. (2007). Development of a view-invariant representation of the human head. Cognition 102, 261-288.

Grill-Spector, K., Henson, R., and Martin, A. (2006). Repetition and the brain: neural models of stimulusspecific effects. Trends Cogn. Sci. 10, $14-23$.

Grill-Spector, K., Kushnir, T., Edelman, S., Avidan, G., Itzchak, Y., and Malach, R. (1999). Differential processing of objects under various viewing conditions in the human lateral occipital complex. Neuron 24, 187-203.

Grill-Spector, K., Kushnir, T., Hendler, T., Edelman, S., Itzchak, Y., and Malach, R. (1998). A Sequence of object-processing stages revealed by fMRI in the human occipital lobe. Hum. Brain Mapp. 6, 316-328.

Grill-Spector, K., and Malach, R. (2001). fMR-adaptation: a tool for studying the functional propertied of human cortical neurons. Acta Psychol. (Amst.) 107, 293-321.

Grossmann, T., Johnson, M. H., Lloyd-Fox, S., Blasi, A., Deligianni, F., Elwell, E., and Csibra, G. (2008). Early cortical specialization for face-to-face communication in human infants. Proc. Biol. Sci. 275, 2803-2811.

Halgren, E., Dale, A. M., Sereno, M. I., Tootell, R. B. H., Marinkovic, K., and Rosen, B. R. (1999). Location of human face-selective cortex with respect to retinotopic areas. Hum. Brain Mapp. 7, 29-37.

Henson, R., Shallice, T., and Dolan, R. (2000). Neuroimaging evidence for dissociable forms of repetition priming. Science 287, 1269-1272.

Henson, R. N. A., and Rugg, M. D. (2003). Neural response suppression, haemodynamic repetition effects, and behavioral priming. Neuropsychologia 41, 263-270.

Honda, Y., Nakato, E., Otsuka, Y., Kanazawa, S., Kojima, S., Yamaguchi, M. K., and Kakigi, R. (2010) How do infants perceive scrambled face? A near-infrared spectroscopic study. Brain Res. 1308, 137-146.

Hoshi, Y., Kobayashi, N., and Tamura, M. (2001). Interpretation of nearinfrared spectroscopy signals: a study with a newly developed perfused rat brain model. J. Appl. Physiol. 90, 1657-1662.

Ichikawa, H., Kanazawa, S., Yamaguchi, M. K., and Kakigi, R. (2010). Infant brain activity while viewing facial movement of point-light displays as measured by near-infrared spectroscopy (NIRS). Neurosci. Lett. 482, 90-94.

Jasper, H. H. (1958). The ten-twenty electrode system of the International Federation. Electroencephalogr. Clin. Neurophysiol. 10, 371-375.

Kanwisher, N., McDermott, J., and Chun, M. M. (1997). The fusiform face area: a module in human extrastriate cortex specialized for face perception. J. Neurosci. 17, 4302-4311.
Kanwisher, N., and Yovel, G. (2006). The fusiform face area: a cortical region specialized for the perception of faces. Philos. Trans. R. Soc. Lond. B Biol. Sci. 361, 2109-2128.

Karen, T., Morren, G., Haensse, D., Bauschatz, A. S., Bucher, H. U., and Wolf, M. (2008). Hemodynamic response to visual stimulation in newborn infants using functional near-infrared spectroscopy. Hum. Brain Mapp. 29, 453-460.

Kobayashi, M., Otsuka, Y., Nakato, E., Kanazawa, S., Yamaguchi, M. K., and Kakigi, R. (2012). Do infants recognize the Arcimboldo images as faces? Behavioral and near-infrared spectroscopic study. J. Exp. Child. Psychol. 111, 22-36.

Kourtzi, Z., and Kanwisher, N. (2001). Representation of perceived object shape by the human lateral occipital complex. Science 293, 1506-1509.

Kusaka, T., Kawada, K., Okubo, K., Nagano, K., Namba, M., Okada, H., Imai, T., Isobe, K., and Itoh, S. (2004). Noninvasive optical imaging in the visual cortex in young infants. Hum. Brain Mapp. 22, 122-132.

Matcher, S. J., Elwell, C. E., Cooper, M., Cope, M., and Delpy, D. T. (1995) Performance comparison of several published tissue near-infrared spectroscopy algorithms. Anal. Biochem. 227, 54-68.

Matsuda, G., and Hiraki, K. (2006). Sustained decrease in oxygenated hemoglobin during video games in the dorsal prefrontal cortex: a NIRS study of children. Neuroimage 29 , 706-711.

Nakato, E., Kanazawa, S., and Yamaguchi, M. K. (2010). Learning unfamiliar faces in infants: the advantage of the regular sequence presentation and the three-quarter view superiority. Jpn. Psychol. Res. 54, 257-267.

Nakato, E., Otsuka, Y., Kanazawa, S. Yamaguchi, M. K., Honda, Y., and Kakigi, R. (2011a). I know this face: neural activity during mother's face perception in 7- to 8-monthold infants as investigated by nearinfrared spectroscopy. Early Hum. Dev. 87, 1-7.

Nakato, E., Otsuka, Y., Kanazawa, S. Yamaguchi, M. K., and Kakigi, R. (2011b), Distinct differences in the pattern of hemodynamic response to happy and angry facial expression in infants - A near-infrared spectroscopic study. Neuroimage 54, 1600-1606.

Nakato, E., Otsuka, Y., Kanazawa, S., Yamaguchi, M. K., Watanabe, S., and Kakigi, R. (2009). When do infants differentiate profile face from frontal face? A near-infrared spectroscopic study. Hum. Brain Mapp. 30, 462-472.

Natu, V. S., Fang, J., Narvekar, A., Keshvari, S., Blanz, V., and O'Toole, A J. (2010). Dissociable neural patterns of facial identity across changes in viewpoint. J. Cogn. Neurosci. 22, 1570-1582.

Otsuka, Y., Nakato, E., Kanazawa, S., Yamaguchi, M. K., Watanabe, S. and Kakigi, R. (2007). Neural activation to upright and inverted faces in infants measured by near infrared spectroscopy. Neuroimage 34, 399-406.

Pena, M., Maki, A., Kovacic, D., Dehaene-Lambertz, G., Koizumi, H., Bouquet, F., and Mehler, J. (2003). Sounds and silence: an optical topography study of language recognition at birth. Proc. Natl. Acad. Sci. U.S.A. 100, 11702-11705.

Pourtois, G., Schwartz, S., Seghier, M. L., Lazeyras, F., and Vuilleumier, P. (2005a). Portrais or people? Distinct representations of face identity in the human visual cortex. J. Cogn. Neurosci. 17, 1043-1057.

Pourtois, G., Schwartz, S., Seghier, M. L., Lazeyras, F., and Vuilleumier, P. (2005b). View-independent coding of face identity in frontal and temporal cortices is modulated by familiarity: an eventrelated fMRI study. Neuroimage 24 , 1214-1224.

Pourtois, G., Schwartz, S., Spiridon, M., Martuzzi, R., and Vuilleumier, P. (2009). Object representation for multiple visual categories overlap in lateral occipital and medial fusiform cortex. Cereb. Cortex 19, 1806-1819.

Puce, A., Allison, T., Asgari, M., Gore, J. C., and McCarthy, G. (1996). Differential sensitivity of human visual cortex to faces, letterstrings, and textures: a functional magnetic resonance imaging study. J. Neurosci. 15 , 5205-5215.

Rose, S. A., Jankowski, J. J., and Feldman, J. F. (2002). Speed of processing and face recognition at 7 and 12 months. Infancy 3, 435-455.

Schroeter, M. L., Zysset, S., Kruggel, F., and von Cramon, D. Y. (2003). Age dependency of the hemodynamic response as measured by functional near-infrared spectroscopy. Neuroimage 19, 555-564.

Shimada, S., and Hiraki, K. (2006). Infant's brain responses to live and televised action. Neuroimage 32, 930-939.

Singh, A. K., and Dan, I. (2006). Exploring the false discovery rate in multichannel NIRS. Neuroimage 32, 930-939. 
Strangman, G., Culver, J. P., Thompson, J. H., and Boas, D. A. (2002). A quantitative comparison of simultaneous BOLD fMRI and NIRS recordings during functional brain activation. Neuroimage 17, 719-731.

Taga, G., and Asakawa, K. (2007). Selectivity and localization of cortical response to auditory and visual stimulation in awake infants aged 2 to 4 months. Neuroimage 26, 1246-1252.

Taga, G., Asakawa, K., Hirasawa, K., and Konishi, Y. (2004). Hemodynamic responses to visual stimulation in occipital and frontal cortex of newborn infants: a near-infrared optical topography study. Pathophysiology 10, 277-281.

Taga, G., Asakawa, K., Maki, A., Konishi, Y., and Koizumi, H. (2003). Brain imaging in awake infants by near-infrared optical topography. Proc. Natl. Acad. Sci. U.S.A. 100, 10722-10727.

Toronov, V., Webb, A., Choi, J. H., Wolf, M., Michalos, A., Gratton, E., and Hueber, D. (2001). Investigation of human brain hemodynamics by simultaneous near-infrared spectroscopy and functional magnetic resonance imaging. Med. Phys. 28, 521-527.

Toronov, V. Y., Zhang, X., and Webb, A. G. (2007). A spatial and temporal comparison of hemodynamic signals measured using optical and functional magnetic resonance imaging during activation in the human primary visual cortex. Neuroimage 34, 1136-1148.

Turati, C., Bulf, H., and Simion, F. (2008). Newborn's face recognition over changes in viewpoint. Cognition 106, 1300-1321.

Turati, C., Sangrigoli, S., Ruel, J., and de Schonen, S. (2004). Evidence of the face inversion effect in 4-month-old infants. Infancy 6, 275-297.

Villringer, A., and Chance, B. (1997). Non-invasive optical spectroscopy and imaging of human brain function. Trends Neurosci. 10, 435-442.

Watanabe, H., Homae, F., and Taga, G. (2010). General specific development of functional activation in the cerebral cortexes of 2- to 3 - month-old infants. Neuroimage 50 1536-1544.

Wilcox, T., Bortfeld, H., Woods, R., Wruck, E., Armstrong, J., and Boas, D. A. (2009). Hemodynamic changes in infant cortex during the processing of featural and spatiotemporal information. Neuropsychologia 47, 657-662.

Wilcox, T., Bortfeld, H., Woods, R., Wruck, E., and Boas, D. A. (2005). Using near-infrared spectroscopy to assess neural activation during object processing in infants. J. Biomed. Opt. 10, 11010.

Wilcox, T., Bortfeld, H., Woods, R. Wruck, E., and Boas, D. A. (2008). Hemodynamic response to featural changes in the occipital and inferior temporal cortex in infants: a preliminary methodological exploration. Dev. Sci. 11, 361-370.

Zevin, J. D., Yang, J., Skipper, J. I., and McCandliss, B. D. (2010). Domain general change detection accounts for "dishabituation" effects in temporal-parietal regions in functional magnetic resonance imaging studies of speech perception. J. Neurosci. 30, 1110-1117.

Conflict of Interest Statement: The authors declare that the research was conducted in the absence of any commercial or financial relationships that could be construed as a potential conflict of interest.

Received: 09 August 2011; accepted: 10 November 2011; published online: 30 November 2011.

Citation: Kobayashi M, Otsuka Y, Nakato E, Kanazawa S, Yamaguchi $M K$ and Kakigi $R$ (2011) Do infants represent the face in a viewpointinvariant manner? Neural adaptation study as measured by near-infrared spectroscopy. Front. Hum. Neurosci. 5:153. doi: 10.3389/fnhum.2011.00153

Copyright (C) 2011 Kobayashi, Otsuka, Nakato, Kanazawa, Yamaguchi and Kakigi. This is an open-access article distributed under the terms of the Creative Commons Attribution Non Commercial License, which permits non-commercial use, distribution, and reproduction in other forums, provided the original authors and source are credited. 